\title{
I. CALCIUM-CARBONATE AND SAND-FRACTION ANALYSIS OF CENOZOIC AND MESOZOIC SEDIMENTS FROM THE MOROCCAN BASIN
}

\author{
Marthe Melguen, Centre Océanologique de Bretagne, BP 337, 29273 Brest Cedex, France
}

\section{INTRODUCTION}

As the DSDP Sites 415 and 416 were essentially drilled to reach deep Mesozoic sedimentary sequences, only very few and spaced Cenozoic cores were recovered (Figures 1 and 2). Consequently it is almost impossible to try to reconstruct in the light of these few cores the evolution of the Cenozoic sedimentary facies off Morocco. Only some major lines of this evolution could be drawn.

Concerning the Mesozoic sediments, while it was easy to measure the calcium carbonate content, it was almost impossible to sieve the sand fraction and, consequently, to study its composition. This is the main reason why the present study is limited to the Cenozoic. Furthermore, samples from Site 370 (Leg 41) were selected in order to complete the Cenozoic sedimentary sequence of Site 416 (Leg 50). The present coarse fraction study is a complementary study to the previous one already done on board the R/V Glomar Challenger during Leg 50. At that time, all the samples selected on board for micropaleontological purposes were also analyzed by the present author in order to appreciate their sand fraction composition and preservation.

\section{ANALYTICAL METHODS}

Samples taken on board the R/V Glomar Challenger were approximately $10 \mathrm{~cm}^{3}$. Calcium carbonate content has been determined ashore for each sample using a Bernard calcimeter. For each sample the sand fraction (63-2000 $\mu \mathrm{m})$ was sieved, dried, and weighed. No $\mathrm{H}_{2} \mathrm{O}_{2}$ was used, to avoid an artificial fragmentation of the planktonic foraminifers. The composition of the total sand fraction was studied under the microscope, and proportions of the different components were visually estimated using the chart of Terry and Chillingar (1955). For this purpose, the sand fraction was a few times divided in sub-fractions and a representative subfraction was put on a plane surface under the microscope. Special attention was given to the preservation of calcareous biogenic components and especially to that of planktonic foraminifers. For each sand fraction the proportion of fragments of tests of planktonic foraminifers was estimated. The degree of fragmentation of planktonic foraminifers observed in the coarse fraction is considered to reflect the degree of carbonate dissolution (Berger, 1970a, 1970b; Melguen and Thiede, 1974). With increasing dissolution the abundance of planktonic foraminifers decreases, while their fragmentation and the abundance of benthic calcareous foraminifers increase (Melguen and Thiede, 1974; Adelseck,
1977). Furthermore, the dissolution of calcareous fossils leads to a relative enrichment of fish debris in the sediments.

\section{RESULTS AND COMMENTS}

Results are presented in Tables 1 through 4.

\section{Site $\mathbf{4 1 5}$}

All data are presented on Figure 1 and in Table 1.

\section{Unit 1}

Nannofossil marl and nannofossil ooze (Cores 415-1, 415-2, 415B-1 and 415B-2; 0 to 110 meters; Pleistocene to upper Miocene.

$\mathrm{CaCO}_{3}$ content: 41 to 70.3 per cent. These values agree perfectly with the facies determination.

Sand content: 6.5 to 17.2 per cent. These values are higher than those deduced from the smear slides observation.

Composition: The sand fraction is essentially a "planktonic-foraminifer sand" containing more than 70 per cent planktonic foraminifers, associated with minor amounts of benthic foraminifers $(\leq 3 \%)$, echinoid spines $(<1 \%)$, radiolarians $(\leq 2 \%)$, ostracodes $(\leq 1 \%)$, sponge spicules $(<1 \%)$, and mollusks $(0.5 \%)$.

Degree of carbonate dissolution: Planktonic foraminifers are very abundant $(70-98 \%)$ and relatively well preserved. The fragmentation varies from 2 to 25 per cent and decreases obviously from core $2(25 \%)$ to core $1(2-10 \%)$, suggesting that carbonate dissolution decreased from late Miocene to Pleistocene times. Furthermore, the presence of common $(>10 \%)$ pteropods at the top of core 415-1 indicates that this site was situated above the $\mathrm{CCO}$ during the Pleistocene.

\section{Unit II}

Alternating marl and nannofossil chalk (Core 415-3; 110 to 199 meters; middle Miocene).

$\mathrm{CaCO}_{3}$ content: 51 to 79 per cent.

Sand content: 2.3 to 11.2 per cent. The sand is probably very fine, because it was considered as silt in the smear slide analysis.

Composition: As in the previous unit, the sand fraction is essentially constituted of planktonic foraminifers $(80-95 \%)$, associated with small amounts of benthic foraminifers $(1-5 \%)$, radiolarians $(\leq 0.5-25 \%)$, sponge spicules $(2-3 \%)$, fish debris $(\leq 1 \%)$, and glauconite $(0.5-1 \%)$. Volcanic glass is common $(\sim 10 \%)$ in $415-3$, $\mathrm{CC}$, and terrigenous minerals are abundant in $4-1$ $(68-70 \%)$. Pyrite is present throughout (1-3\%). 


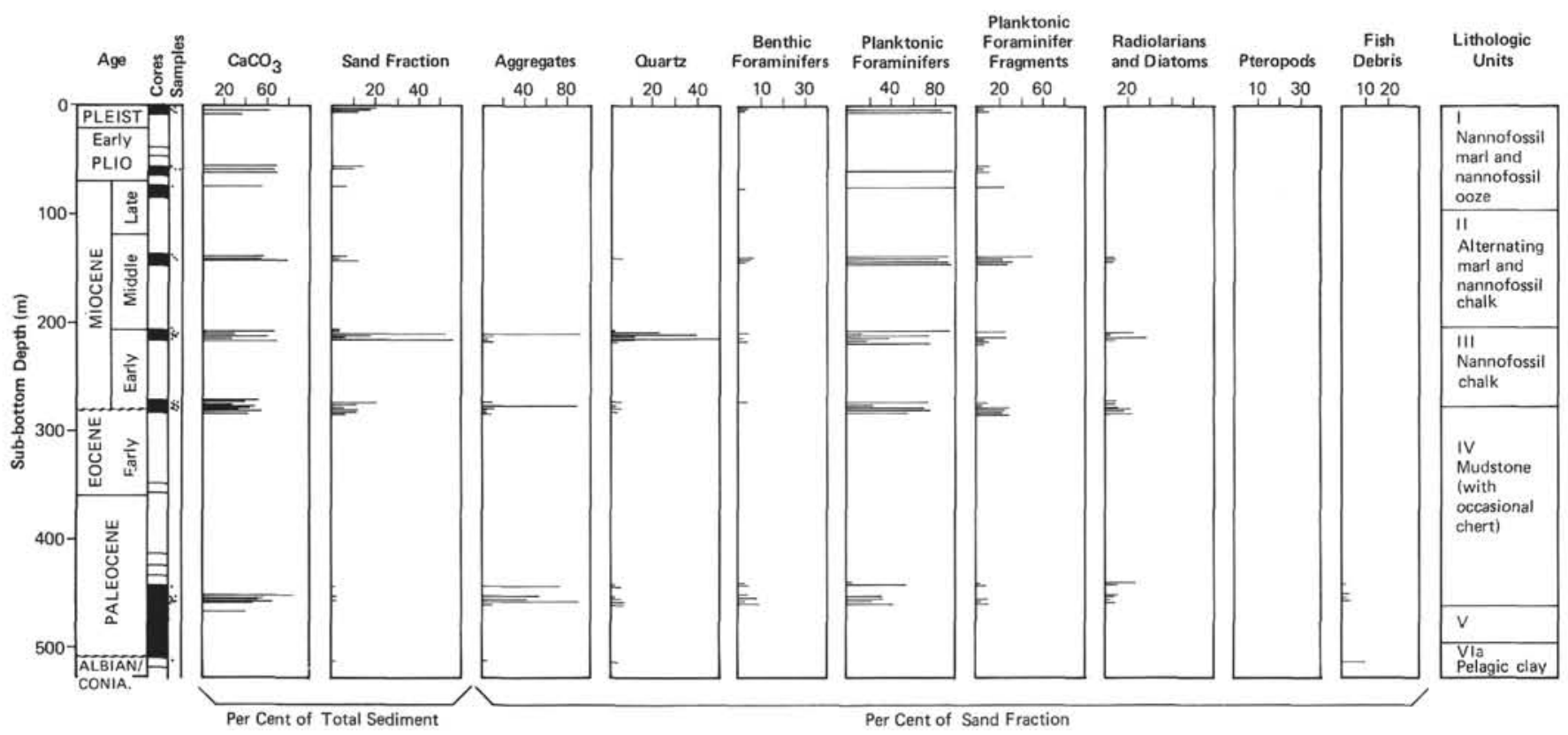

Figure 1. Variations in carbonate content and sand-fraction content with respect to total sediment at Site 415. Also shown are variations in the most-significant terrigenous and biogenic components in the sand fraction (63-2000 $\mu \mathrm{m})$ and variations in the degree of fragmentation of the planktonic foraminifers.

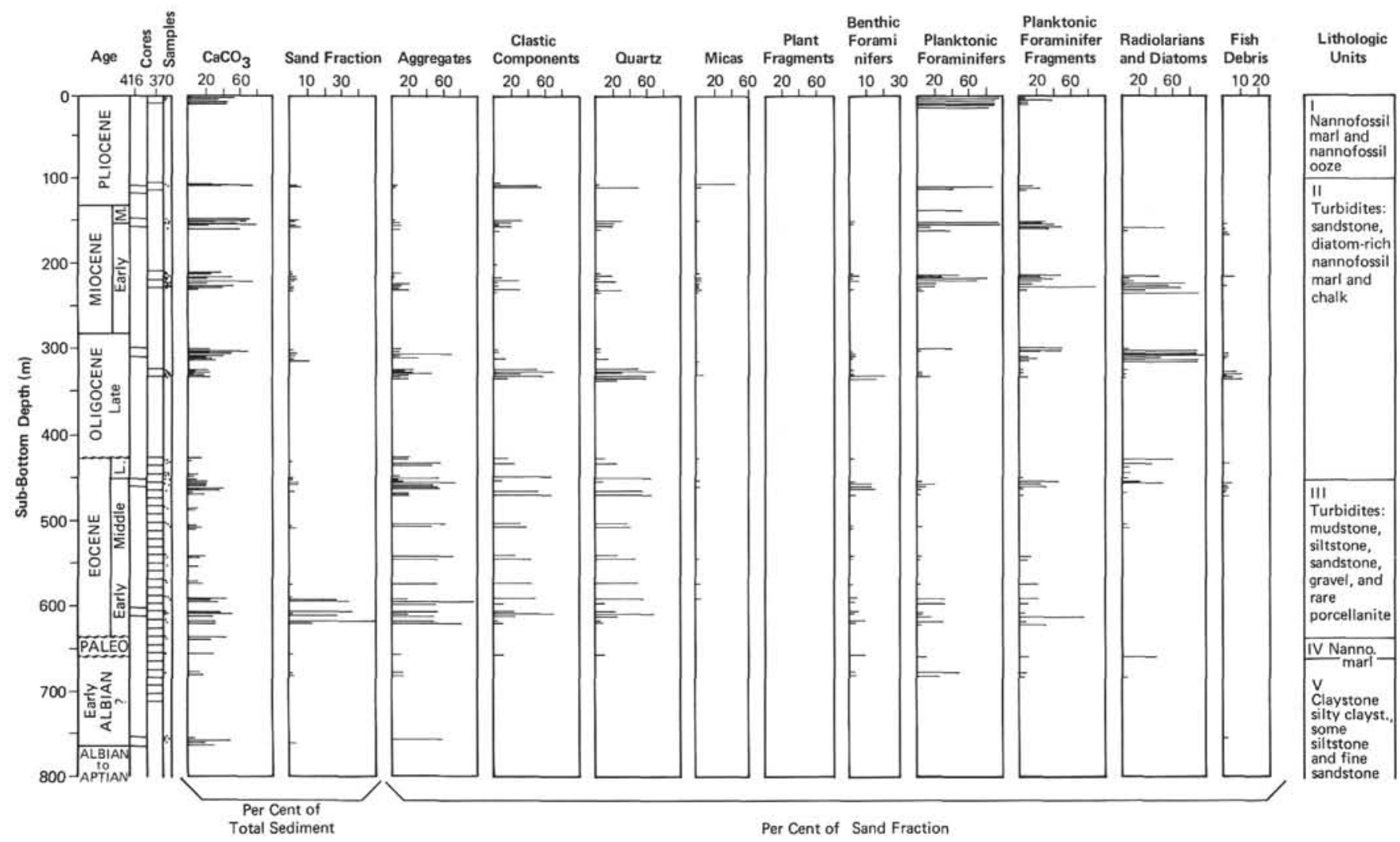

Figure 2. Variations in carbonate content and sand-fraction content with respect to total sediment at Site 416 and Site 370. Also shown are variations in the most-significant terrigenous and biogenic components in the sand fraction (63-2000 $\mu \mathrm{m})$ and variations in the degree of fragmentation of the planktonic foraminifers. 
TABLE 1

\begin{tabular}{|c|c|c|c|c|c|c|c|c|c|c|c|c|c|c|c|c|c|c|}
\hline \multirow[b]{2}{*}{$\begin{array}{c}\begin{array}{c}\text { Sample } \\
\text { (interval in } \mathrm{cm} \text { ) }\end{array} \\
\end{array}$} & \multicolumn{2}{|c|}{$\begin{array}{l}\text { Total Sediment } \\
\text { Content }(\%)\end{array}$} & \multicolumn{16}{|c|}{ Sand Fraction $(>63 \mu \mathrm{m})$ Content $(\%)$} \\
\hline & $\mathrm{CaCO}_{3}$ & $\begin{array}{c}\text { Sand } \\
\text { Fraction }\end{array}$ & $\begin{array}{l}\text { Aggre- } \\
\text { gates }\end{array}$ & Quartz & Micas & Pyrite & $\begin{array}{l}\text { Glauco- } \\
\text { nite }\end{array}$ & $\begin{array}{l}\text { Radio- } \\
\text { larians }\end{array}$ & Diatoms & $\begin{array}{l}\text { Siliceous } \\
\text { Spicules }\end{array}$ & $\begin{array}{c}\text { Fish } \\
\text { Debris }\end{array}$ & $\begin{array}{c}\text { Benthic } \\
\text { Foraminifers }\end{array}$ & $\begin{array}{c}\text { Planktonic } \\
\text { Foraminifers }\end{array}$ & $\begin{array}{l}\text { Planktonic } \\
\text { Foraminifer } \\
\text { Fragments }\end{array}$ & $\begin{array}{l}\text { Echi- } \\
\text { noids }\end{array}$ & Bivalves & $\begin{array}{c}\text { Plant } \\
\text { Fragments }\end{array}$ & $\begin{array}{c}\text { Volcanic } \\
\text { Glass }\end{array}$ \\
\hline $415-1-1,80-82$ & 58.5 & 17.2 & & & & & & & & & & 3 & 70 & $<2$ & & & & \\
\hline $1-1,82-84$ & 57.0 & 13.6 & & & & & & & & & & 2 & $>85$ & $<2$ & & TR & & \\
\hline $1-1,120-122$ & 50.5 & 14.4 & & & TR & & & & & TR & & $\leqslant 2$ & $>95$ & 5 & TR & & & \\
\hline $1-4,130-132$ & 41.0 & 13.0 & & & & & & & & & & $<2$ & 98 & $<10$ & & & & \\
\hline $1-5,102-104$ & 57.5 & 12.9 & & & & & & & & & TR & $<2$ & 98 & $<10$ & & & & \\
\hline $2-1,93-96$ & 58.5 & 6.5 & & & & 3 & & 1 & & & 1 & $\leqslant 2$ & 95 & 25 & & & & \\
\hline $3-1,60-62$ & 65.5 & 2.3 & & TR & & 1 & 1 & 3 & & 2 & 1 & 5 & 90 & 50 & $\mathrm{TR}$ & & & \\
\hline $3-2,123-125$ & 59.0 & 7.6 & 2 & 5 & $\mathrm{TR}$ & 1 & 1 & 5 & & 1 & & 3 & 80 & 25 & 0.5 & & & \\
\hline $3-3,50-52$ & 54.5 & 4.2 & 5 & 2 & & 3 & TR & 3 & & 1 & & 2 & 85 & 30 & 0.0 & & & \\
\hline $3-3,80-82$ & 79.0 & 11.2 & 2 & $\mathrm{TR}$ & & 3 & 0.5 & $\mathrm{TR}$ & & TR & 1 & 1 & 95 & 25 & & & & \\
\hline $4-1,32-34$ & 69.5 & 3.0 & TR & 1 & & $\leqslant 1$ & & TR & & 1 & & 1 & 95 & 25 & $\mathrm{TR}$ & & & \\
\hline $4-1,68-70$ & 51.0 & 3.9 & 15 & 40 & TR & 3 & 1 & 25 & & TR & 1 & 3 & 10 & & $\mathrm{TR}$ & & & \\
\hline $4-2,30-32$ & 29.0 & 54.3 & 100 & & & & & & & & & & & & & & & \\
\hline $4-2,70-72$ & 62.3 & 16.8 & 3 & 10 & & & & 1 & & 1 & & $\varsigma 1$ & 75 & $<25$ & & & & $<10$ \\
\hline $4-5,54-56$ & 25.0 & 3.4 & 10 & 50 & $\mathrm{TR}$ & 3 & & 35 & 1 & 2 & & & 5 & $<5$ & & & & \\
\hline $4-5,102-103$ & 69.5 & 54.1 & 35 & 10 & & 2 & 2 & 5 & 1 & & & 2 & 10 & $<10$ & 2 & 2 & & \\
\hline $4-5,106-108$ & 73.5 & 11.2 & 10 & 3 & & 2 & $\mathrm{TR}$ & 5 & 1 & & 2 & 2 & 75 & $\leqslant 5$ & 1 & 2 & & \\
\hline $5-1,30-32$ & 52.0 & 13.0 & 10 & 5 & & 2 & & $\leqslant 5$ & 3 & & 1 & $\leqslant 2$ & 70 & $<10$ & & & & \\
\hline $5-1,100-102$ & 38. & 19.9 & 90 & & & & & 5 & 1 & TR & TR & 2 & 3 & $<2$ & & & & \\
\hline $5-3,50-52$ & 27.0 & 11.8 & 2 & 10 & & 20 & 3 & 7 & 1 & 2 & 0.5 & 3 & 50 & $<5$ & $\mathrm{TR}$ & & & \\
\hline $5-4,65-67$ & 48.0 & 6.8 & 10 & 3 & TR & 10 & & $<5$ & 2 & & TR & 2 & 70 & 30 & TR & & & \\
\hline $5-4,67-69$ & 46.5 & 5.8 & 5 & 2 & & 5 & & 15 & 5 & & 1 & 2 & 65 & $<25$ & & & & \\
\hline $5-6,25-27$ & 55. & 11.2 & 5 & 5 & & 2 & & 10 & 2 & & 1 & 2 & 75 & $<25$ & & & & \\
\hline $5-6,41-43$ & 43. & 6.1 & 10 & 3 & & 15 & & 15 & 7 & & 1 & & 50 & 30 & & & & \\
\hline $415 \mathrm{~A}-5-1,129-131$ & 2.5 & 1.2 & 75 & 1 & & TR & & 26 & 0.5 & TR & & & 0.5 & & & & & \\
\hline $6-1,72-74$ & 84. & 2.2 & 55 & 1 & & & & 5 & 1 & 2 & 5 & & 30 & 5 & & & & \\
\hline $6-1,110-112$ & 55. & 0.7 & 40 & 5 & & 2 & 3 & 5 & & 1 & 15 & & 30 & 5 & 2 & & & \\
\hline $6-2,8-10$ & 49. & 1.6 & 90 & 5 & & & & & & & 3 & & 2 & & & & & \\
\hline $6-2,37-39$ & 63.5 & 0.9 & 5 & 5 & & 30 & & 5 & 1 & 3 & 15 & & 35 & 5 & 2 & & & \\
\hline $7-1,26-28$ & 3. & 0.1 & 5 & 3 & 1 & & & 80 & TR & 10 & 2 & & TR & 100 & & & & \\
\hline $9-1,92-94$ & 50.4 & 0.3 & 30 & & & & & 10 & & 10 & 2 & & 50 & 2 & & & & \\
\hline $9-3,76-78$ & 34.4 & 0.3 & 20 & & & & & 10 & & 20 & 2 & & 50 & 2 & & & & \\
\hline $11-1,6-8$ & 28.0 & 0.9 & 60 & & & 3 & & 5 & & & & & 30 & 2 & & & & \\
\hline $11, \mathrm{CC} 5-7$ & 28.8 & 5.7 & 25 & & & 2 & & 25 & & 3 & 5 & & 40 & 2 & & & & \\
\hline $12-1,111-113$ & $\begin{array}{l}27.6 \\
27.2\end{array}$ & 5.5 & & & & 1 & & 3 & & 3 & 0.5 & & 2 & 2 & 1 & 70 & & \\
\hline $12-2,113-115$ & 25.6 & 5.6 & 35 & & & 3 & & 45 & & & & & 15 & 2 & & & & \\
\hline $13-1,128-130$ & 56.8 & 9.1 & & & & & & 45 & & & & & 15 & 2 & & & & \\
\hline $14-1,25-27$ & 34.4 & 0.2 & 50 & 2 & 1 & 3 & & 2 & & 3 & 2 & & 20 & 2 & & 10 & & \\
\hline $415 \mathrm{~B}-2-1,42-48$ & 69.5 & 14.6 & & & & 0.5 & & & & TR & 2 & & 97 & 10 & 1 & & & \\
\hline $2-1,120-122$ & 67.9 & 10.5 & & & & TR & & & & TR & 2 & & 97 & $\begin{array}{r}100 \\
5\end{array}$ & 0.5 & 0.5 & & \\
\hline $2-2,70-72$ & 70.3 & 9.6 & & 1 & & & & & & 1 & 2 & & 95 & 10 & 0.5 & & & \\
\hline
\end{tabular}


TABLE 2

Site 416

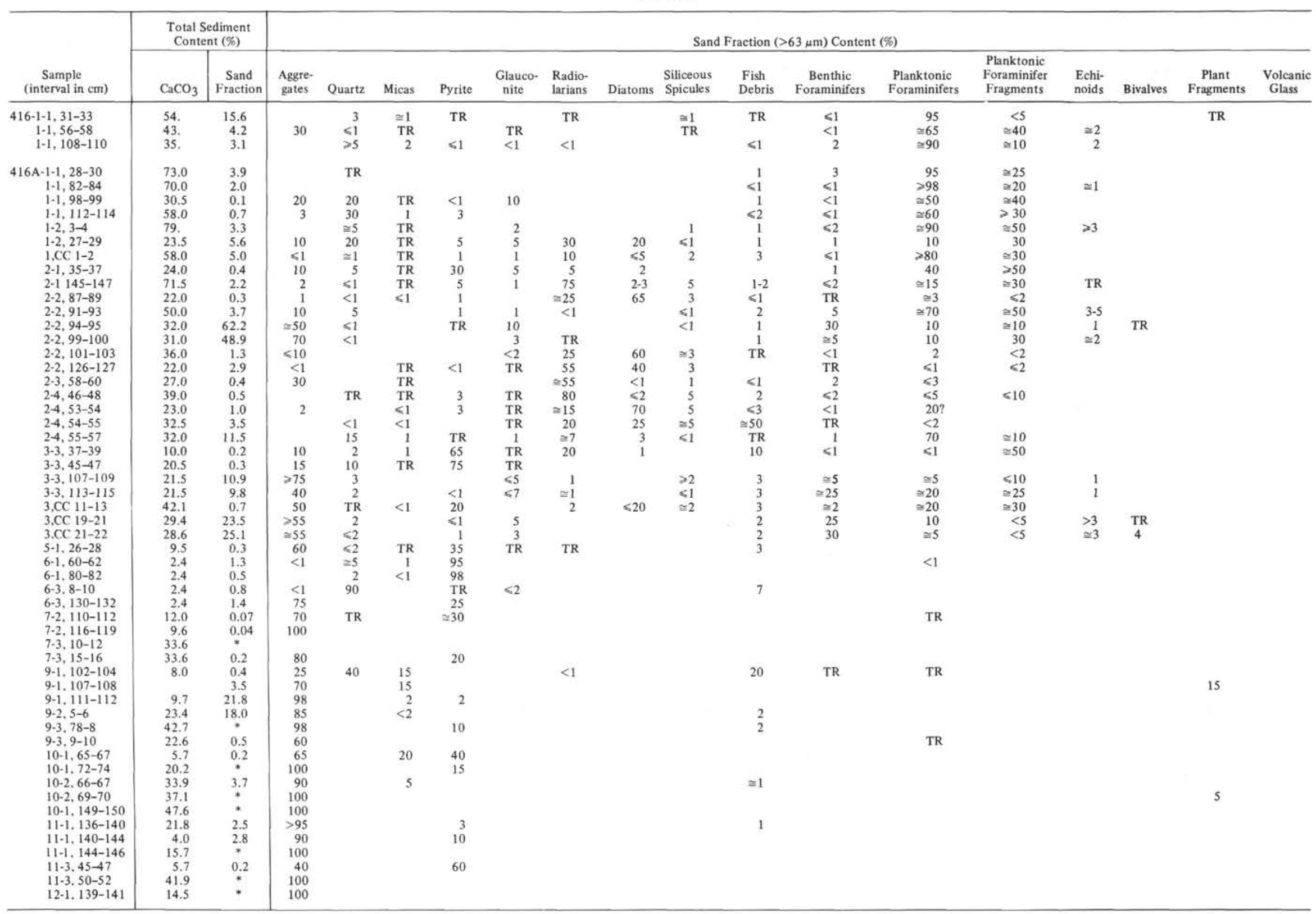


TABLE 3

Site 370

\begin{tabular}{|c|c|c|c|c|c|c|c|c|c|c|c|c|c|c|c|c|c|c|}
\hline \multirow[b]{2}{*}{$\begin{array}{c}\text { Sample } \\
\text { (interval in cm) }\end{array}$} & \multicolumn{2}{|c|}{$\begin{array}{l}\text { Total Sediment } \\
\text { Content }(\%)\end{array}$} & \multirow[b]{2}{*}{$\begin{array}{l}\text { Aggre- } \\
\text { gates }\end{array}$} & \multirow[b]{2}{*}{ Quartz } & \multirow[b]{2}{*}{ Micas } & \multirow[b]{2}{*}{ Pyrite } & \multirow[b]{2}{*}{$\begin{array}{c}\text { Glauco- } \\
\text { nite }\end{array}$} & \multirow[b]{2}{*}{$\begin{array}{l}\text { Radio- } \\
\text { larians }\end{array}$} & \multirow[b]{2}{*}{ Diatoms } & Sand & raction ( & $63 \mu \mathrm{m})$ Content & & & & & & \\
\hline & $\mathrm{CaCO}_{3}$ & \begin{tabular}{|c|} 
Sand \\
Fraction
\end{tabular} & & & & & & & & $\begin{array}{l}\text { Siliceous } \\
\text { Spicules }\end{array}$ & $\begin{array}{c}\text { Fish } \\
\text { Debris }\end{array}$ & $\begin{array}{c}\text { Benthic } \\
\text { Foraminifers }\end{array}$ & $\begin{array}{c}\text { Planktonic } \\
\text { Foraminifers }\end{array}$ & $\begin{array}{c}\text { Planktonic } \\
\text { Foraminifer } \\
\text { Fragments }\end{array}$ & $\begin{array}{l}\text { Echi- } \\
\text { noids }\end{array}$ & Bivalves & $\begin{array}{c}\begin{array}{c}\text { Plant } \\
\text { Fragments }\end{array} \\
\end{array}$ & $\begin{array}{c}\text { Volcanic } \\
\text { Glass } \\
\end{array}$ \\
\hline $370-1-2,50-52$ & 32.8 & 2.9 & $\approx 5$ & $\approx 10$ & & & TR & & & & & $<2$ & 85 & $<5$ & $\approx 0.5$ & & & \\
\hline $1-2,100-102$ & 45.9 & 3.6 & $\cong 10$ & 1 & & TR & & & & & TR & $\approx 1$ & 90 & $\cong 10$ & thos & & & \\
\hline $1-4,50-52$ & 42.3 & 4.2 & 10 & 15 & 2 & 3 & 2 & & & & 1 & 2 & 65 & $<25$ & & & & \\
\hline $1-4,120-122$ & 44.7 & 3.1 & 2 & To & 0.5 & 1 & $<1$ & & & & 1 & 3 & 90 & $\cong 25$ & & & & \\
\hline $1-5,50-52$ & 45.9 & 3.9 & 10 & 0.5 & & $<0.5$ & 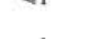 & & & & 0.5 & $<1$ & $\approx 90$ & $\cong 10$ & $\approx 0.5$ & & & \\
\hline $2-1,80-82$ & 48.4 & 3.5 & 0.5 & 1 & & TR & 1 & & & & TR & 2 & & $\approx 15$ & $\cong 1$ & & & \\
\hline $2-2,10-12$ & 76.4 & 4.7 & & & & & 1 & & & & 1 & $\leqslant 2$ & 95 & $\cong 25$ & & & & \\
\hline $2-2,100-102$ & 34.4 & 5.4 & 2 & 50 & 5 & & 3 & & & & & $<1$ & 40 & $<5$ & & & & \\
\hline $2-2,140-142$ & 26.0 & 0.1 & $\leqslant 5$ & 3 & 45 & 2 & & & & & 3 & 0.5 & 40 & $\cong 30$ & & & & \\
\hline $3-1,50-52$ & 38.5 & 0.4 & 10 & 5 & 2 & $\cong 30$ & 2 & & & & $\leqslant 1$ & 2 & $\cong 50$ & $\cong 50$ & & & & \\
\hline $3-1,130-132$ & 26.2 & 0.3 & 2 & 20 & 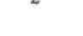 & 20 & 2 & 45 & & & $\geqslant 5$ & 5 & 3 & 25 & & & & \\
\hline $3-2,50-52$ & 52.5 & 2.1 & $i$ & & & 2 & $i$ & $\leqslant 10$ & 0.5 & & 1 & 2 & $>80$ & $\approx 40$ & & & & \\
\hline $3-2,60-62$ & 50.4 & 3.2 & $\leqslant 1$ & 3 & $\leqslant 1$ & $i$ & 2 & 15 & 1 & 3 & 1 & 1 & 70 & 20 & & & - & \\
\hline $3-2,88-90$ & 17.9 & 1.5 & 10 & 25 & 2 & 2 & 1 & 30 & 3 & 5 & 1 & 5 & 20 & 10 & & & & \\
\hline $3-2,118-120$ & 76.4 & 2.6 & 5 & 1 & $<1$ & 0.5 & & $\leqslant 30$ & $\leq 1$ & 5 & 2 & $\leqslant 1$ & $>50$ & $\cong 10$ & & & & \\
\hline $4-2,50-52$ & 21.3 & 0.9 & 20 & . & $\mathrm{TR}$ & 2 & & 45 & 30 & & 2 & . & 1 & $=10$ & & & & \\
\hline $4-2,66-68$ & 20.3 & 0.3 & 5 & 3 & 3 & 15 & 0.5 & 30 & 30 & 10 & 0.5 & & 1 & $<5$ & & & & \\
\hline $4-2,70-73$ & 16.4 & 0.7 & 2 & 1 & 2 & 10 & & 35 & 20 & 然 & & & & & & & & \\
\hline $4-2,111-113$ & 53.7 & 0.5 & 2 & 3 & 2 & 15 & 0.5 & 50 & 1 & $<5$ & 2 & 1 & 20 & 90 & & & & \\
\hline $4-3,20-22$ & 5.7 & 1.0 & 10 & 3 & 2 & 3 & & 60 & 10 & 3 & & 2 & 1 & & & & & \\
\hline $4-3,100-102$ & 31.2 & 0.7 & 7 & $\leqslant 1$ & $\leqslant 1$ & $\cong 20$ & 0.5 & 20 & $\leqslant 40$ & 2 & TR & & & & & & & \\
\hline $4-4,14-16$ & 25.2 & 0.4 & 5 & & 0.5 & 5 & 0.5 & 20 & 70 & 5 & 0.5 & & & & & & & \\
\hline $4-4,38-40$ & 38.2 & 1.0 & 1 & 1 & 0.5 & 3 & 0.5 & 75 & 5 & 10 & 1 & 2 & & & & & & \\
\hline $4-4,120-122$ & 21.3 & 0.9 & 20 & 30 & 2 & 5 & 3 & 20 & 10 & & . & 2 & 5 & 10 & & & & \\
\hline $5-1,120-122$ & 21.3 & 0.6 & 25 & 50 & & 10 & 2 & $\leqslant 5$ & 1 & 1 & TR & 2 & 2 & $<2$ & & & & \\
\hline $5-2,20-22$ & 6.6 & 0.3 & 15 & 70 & & & $<5$ & TR & & 2 & 7 & $\mathrm{TR}$ & 1 & $\leqslant 2$ & & & & \\
\hline $5-2,100-102$ & 18.9 & 0.4 & 45 & 10 & 1 & 40 & & 2 & & $i$ & & $\mathrm{TR}$ & & & & & & \\
\hline $5-3,10-12$ & 4.1 & 0.3 & & $\cong 30$ & & 55 & 1 & $\mathrm{TR}$ & & 2 & 10 & & 2 & $<2$ & & & & \\
\hline $5 \cdot 3,60-62$ & 25.2 & 0.2 & 15 & 40 & 0.5 & 0.5 & 15 & & 2 & & 0.5 & 0.5 & 25 & 2 & $\leqslant 2$ & & & \\
\hline $5-3,105-107$ & 4.9 & 0.2 & 2 & 50 & 2 & 25 & 2 & 3 & & 3 & 10 & 1 & 2 & 10 & & & & \\
\hline $5-3,130-132$ & 14.8 & 0.5 & 20 & 60 & 5 & 10 & TR & TR & & TR & & 1 & $\geqslant 5$ & & & & & \\
\hline $5-4,20-22$ & 19.7 & 7.6 & 15 & 10 & & & 30 & & & & 5 & 20 & 15 & $<10$ & $\cong 5$ & $\cong 5$ & & \\
\hline $5-5,30-32$ & 3.3 & 1.0 & 5 & 60 & & & 25 & $\mathrm{~T}$ & & & 10 & & TR & & & & & \\
\hline $5-6,130-132$ & 9.0 & 0.2 & 20 & 15 & & 30 & 5 & 2 & & 3 & 10 & 15 & & & & & & \\
\hline $6-2,50-52$ & 2.5 & 0.9 & 55 & 25 & 2 & 15 & $\leqslant 1$ & 10 & & 3 & 3 & 0.5 & 0.5 & $\leqslant 2$ & & & & \\
\hline $6-2,101-103$ & 4.1 & 0.01 & 20 & 10 & 2 & 3 & 2 & 60 & 1 & & i & 1 & & & & & & \\
\hline $6-2,140-142$ & 3.2 & 4.4 & 50 & 3 & & 2 & 5 & 35 & 1 & & 0.5 & 1 & & & & & & \\
\hline $7-2,40-42$ & 10.6 & 4.8 & 60 & 30 & 0.5 & 1 & 1 & 5 & & & 0.5 & 0.5 & 0.5 & & 0.5 & & & \\
\hline $7-2,120-122$ & 6.7 & 1.5 & 10 & 70 & & 5 & 2 & 7 & & & & 0.5 & 3 & $<2$ & & & & \\
\hline $7-2,128-130$ & 5.7 & 1.0 & 5 & 30 & & 15 & 0.5 & 50 & & & 0.5 & & 0.5 & & & & & \\
\hline $8-1,129-131$ & 4.9 & 2.1 & 20 & 55 & & 20 & 0.5 & 5 & & & & & & & & & & \\
\hline $8-2,112-115$ & 20.5 & 3.9 & 20 & 65 & & 5 & 1 & 2 & & & 2 & 3 & 3 & $\leqslant 5$ & 1 & 1 & & \\
\hline $10-2,114-116$ & 22.8 & 2.4 & 60 & 35 & & & 1 & 2 & & & 0.5 & 1 & 1 & $\leqslant 10$ & & & & \\
\hline $10-2,118-120$ & 11.5 & 1.4 & so & 40 & & 2 & & 5 & & & & 1 & 2 & & & & & \\
\hline $10-2,125-127$ & 17.1 & 1.0 & 60 & 35 & & 2 & & 2 & & & & 1 & 1 & & & & & \\
\hline $12-2,18-20$ & 21.1 & 36.6 & 70 & 25 & & 0.5 & 1 & i & & & 0.5 & 1 & $<2$ & $\leqslant 10$ & & & & \\
\hline $12-2,134-136$ & 13.8 & 24.3 & so & 45 & & & 2 & 1 & & & . & 1 & 1 & $<10$ & & & & \\
\hline $14-2,25-28$ & 17.9 & 0.9 & so & 45 & 0.5 & 2 & 0.5 & 0.5 & & & & 0.5 & 1 & $\leqslant 20$ & & & & \\
\hline $15-3,19-22$ & 46.3 & 0.5 & 20 & 45 & 0.5 & & $<3$ & & & & 0.5 & 3 & 30 & $\leqslant 20$ & & & & \\
\hline $15-3,23-25$ & 27.7 & 25.8 & 50 & $\leqslant 25$ & 0.5 & 0.5 & 2 & & & & 3 & 5 & 10 & $\leqslant 10$ & & & & \\
\hline $15-3,127-129$ & 35.8 & 34.6 & 55 & 10 & & 0.5 & 0.5 & & & & 3 & $<2$ & $\leqslant 30$ & $\leqslant 10$ & & & & \\
\hline $16-2,16-20$ & 36.6 & 33.2 & 55 & 25 & & & 3 & & & & 3 & 10 & 2 & $<5$ & & & & \\
\hline $16-2,39-41$ & 51.2 & 0.5 & 20 & $\leqslant 70$ & & & $<5$ & & & & 3 & $<5$ & 2 & & & & & \\
\hline $16-2,57-60$ & 32.5 & 26.8 & so & 25 & 1 & & & & & & 2 & 10 & 15 & 75 & & & & \\
\hline $17-2,50-53$ & 33.3 & 14.0 & 50 & 3 & & & & & & & 0.5 & 15 & 30 & $<10$ & & & & \\
\hline $17-2,137-140$ & 32.5 & 51.5 & 85 & 10 & & & 1 & & & & 1 & 3 & 1 & 30 & & & & \\
\hline $19-1,116-118$ & 30.9 & 1.0 & 10 & 10 & & & 2 & 40 & & & 1 & 15 & $\leqslant 10$ & $\leqslant 10$ & & & & \\
\hline $20-2,39-42$ & 13.0 & 0.6 & 10 & 0.5 & & & & & & & 1 & 1 & 50 & $<5$ & & & & \\
\hline $20-2,140-142$ & 16.3 & 0.6 & 10 & & & & & 0.5 & & & & 2 & 25 & $<5$ & & & & \\
\hline
\end{tabular}


TABLE 4

Hole 416A

\begin{tabular}{|c|c|c|}
\hline \multirow[b]{2}{*}{$\begin{array}{c}\text { Core/Section/Level } \\
\text { Sampled }(\mathrm{cm})\end{array}$} & \multicolumn{2}{|c|}{$\begin{array}{l}\text { Total Sediment } \\
\text { Content }(\%)\end{array}$} \\
\hline & $\mathrm{CaCO}_{3}$ & $\begin{array}{c}\text { Sand } \\
\text { Fraction }\end{array}$ \\
\hline $\begin{array}{c}416 \mathrm{~A}-12-4,125-127 \\
12-4,135-138 \\
13-2,65-67 \\
13-2,72-74 \\
13-2,74-75\end{array}$ & $\begin{array}{r}7.3 \\
24.2 \\
15.3 \\
8.9 \\
22.6\end{array}$ & $\begin{array}{c}* \\
* \\
0.2 \\
11.6 \\
*\end{array}$ \\
\hline $\begin{array}{l}14-1,103-105 \\
14-1,109-111 \\
14-1,112-113 \\
14-2,116-117 \\
14-5,2-3\end{array}$ & $\begin{array}{r}13.7 \\
3.2 \\
2.4 \\
15.3 \\
6.4\end{array}$ & $\begin{array}{l}0.1 \\
0.1 \\
0.3 \\
2.0 \\
0.05\end{array}$ \\
\hline $\begin{array}{l}14-5,8-10 \\
14-5,25-29 \\
14, \text { CC } 28 \\
16-1,30-31 \\
16-1,34-35\end{array}$ & $\begin{array}{r}7.3 \\
32.3 \\
9.7 \\
10.5 \\
8.0\end{array}$ & $\begin{array}{c}1.4 \\
0.9 \\
50.6 \\
* \\
0.07\end{array}$ \\
\hline $\begin{array}{l}16-1,118-120 \\
16-1,124-125 \\
16-1,137-138 \\
16-2,91-92 \\
18-1,117-118\end{array}$ & $\begin{array}{l}35.5 \\
65.3 \\
35.5 \\
28.2 \\
25.0\end{array}$ & $\begin{array}{c}0.02 \\
0.1 \\
26.0 \\
* \\
0.5\end{array}$ \\
\hline $\begin{array}{l}18-1,118-119 \\
18-1,120-122 \\
18-1,122-124 \\
18-1,125-127 \\
18-1,139-141\end{array}$ & $\begin{array}{r}8.1 \\
4.0 \\
6.5 \\
25.0 \\
36.3\end{array}$ & $\begin{array}{l}0.1 \\
0.02 \\
0.3 \\
* \\
0.5\end{array}$ \\
\hline $\begin{array}{l}19-1,40-42 \\
19-1,46-47 \\
19-2,90-92 \\
19-2,99-101 \\
19-2,107-109\end{array}$ & $\begin{array}{r}4.0 \\
10.5 \\
5.7 \\
44.4 \\
55.6\end{array}$ & $\begin{array}{c}0.3 \\
0.03 \\
0.04 \\
109 \\
*\end{array}$ \\
\hline $\begin{array}{l}19-2,110-112 \\
20-1,94-96 \\
20-1,104-106 \\
20-1,105-107 \\
20-3,0-2\end{array}$ & $\begin{array}{l}32.3 \\
3.23 \\
9.7 \\
16.3 \\
14.6\end{array}$ & $\begin{array}{l}5.7 \\
0.03 \\
0.03 \\
0.3 \\
0.04\end{array}$ \\
\hline $\begin{array}{l}20-3,20-22 \\
20-3,70-72 \\
21-1,2-4 \\
21-1,24-25 \\
21-1,26-28\end{array}$ & $\begin{array}{l}23.6 \\
52.0 \\
49.6 \\
59.4 \\
41.5\end{array}$ & $\begin{array}{l}0.04 \\
* \\
* \\
* \\
*\end{array}$ \\
\hline $\begin{array}{l}21-1,34-36 \\
23-2,11-13 \\
25-1,82-84 \\
25-1,101-102 \\
25-1,105-106\end{array}$ & $\begin{array}{r}37.4 \\
39.0 \\
3.3 \\
4.1 \\
3.3\end{array}$ & $\begin{array}{l}* \\
0.2 \\
0.1 \\
0.5 \\
*\end{array}$ \\
\hline $\begin{array}{l}25-1,112-113 \\
25-1,117-118 \\
27-4,109-111 \\
29-5,12-14 \\
29-5,25-27\end{array}$ & $\begin{array}{r}40.7 \\
33.3 \\
17.1 \\
4.9 \\
8.9\end{array}$ & $\begin{array}{l}21.0 \\
1.6 \\
0.02 \\
0.4 \\
*\end{array}$ \\
\hline $\begin{array}{l}30-3,57-60 \\
30-3,130-131 \\
30-3,138-139 \\
30-3,139-141 \\
30-5,69-70\end{array}$ & $\begin{array}{r}4.1 \\
36.6 \\
38.2 \\
35.0 \\
48.0\end{array}$ & $\begin{array}{r}1.2 \\
1.0 \\
10.4 \\
* \\
11.9\end{array}$ \\
\hline $\begin{array}{l}30-5,70-71 \\
36-3,4-6 \\
36-3,12-14 \\
37-2,33-34 \\
37-2,37-38\end{array}$ & $\begin{array}{l}40.7 \\
48.0 \\
57.7 \\
50.4 \\
47.1\end{array}$ & $\begin{array}{l}* \\
* \\
* \\
* \\
*\end{array}$ \\
\hline
\end{tabular}

TABLE 4 - Continued

\begin{tabular}{l|rc}
\hline & \multicolumn{2}{|c}{$\begin{array}{c}\text { Total Sediment } \\
\text { Content (\%) }\end{array}$} \\
\cline { 2 - 3 } $\begin{array}{c}\text { Core/Section/Level } \\
\text { Sampled (cm) }\end{array}$ & $\mathrm{CaCO}_{3}$ & $\begin{array}{c}\text { Sand } \\
\text { Fraction }\end{array}$ \\
\hline $37-2,51-53$ & 8.1 & $*$ \\
$37-3,86-87$ & 27.6 & 2.9 \\
$37-3,88-89$ & 20.3 & $*$ \\
$37-4,6-7$ & 6.5 & 0.03 \\
$38-1,22-24$ & 11.4 & 0.03 \\
$38-1,108-109$ & 7.3 & 6.7 \\
$43-1,42-44$ & 4.1 & 0.03 \\
$43-2,1-3$ & 62.6 & $*$ \\
$43-3,149-151$ & 16.3 & 0.02 \\
$43, \mathrm{CC}$ & 15.5 & $*$ \\
$44-1,134-138$ & 12.2 & 0.7 \\
$46-4,36-37$ & 8.2 & 0.1 \\
$46-4,42-44$ & 17.9 & 0.1 \\
$46-4,49-50$ & 29.3 & $*$ \\
$48-1,85-88$ & 37.4 & $*$ \\
$48-1,93-95$ & 4.1 & 0.2 \\
$50-2,0-2$ & 3.3 & 0.4 \\
$50-2,102-104$ & 24.4 & 0.1 \\
$50-2,117-119$ & 78.9 & $*$ \\
$53-2,41-45$ & 4.9 & $*$ \\
$53-2,139-141$ & 6.5 & 1.1 \\
$57-1,109-110$ & 6.5 & 0.4 \\
\hline & & \\
\hline
\end{tabular}

Degree of carbonate dissolution: Planktonic foraminifers are generally very abundant $(\geq 80 \%)$ throughout this unit. Their fragmentation is higher $(25-50 \%)$ than in unit $\mathrm{I}$, which probably reflects greater carbonate dissolution, which could be related to higher oceanic productivity.

\section{Unit III}

Alternating nannofossil chalk and siliceous-debrisbearing marl (Cores 415-4 and 415-5; 199 to 313 meters; middle and lower Miocene).

$\mathrm{CaCO}_{3}$ content: 25 to 73.5 per cent.

Sand content: 3.4 to 54.1 per cent.

Composition: Throughout this unit the sand fraction is alternately rich in biogenic particles (planktonic foraminifers or radiolarians) or terrigenous particles (quartz). The chalky layers contain 75 per cent planktonic foraminifers, while the marly layers contain only 3 to 10 per cent planktonic foraminifers, but as much as 50 per cent quartz, 35 per cent radiolarians and from 1 to 7 per cent diatoms. The relatively high abundance of biogenic siliceous particles might be related to an increase in oceanic fertility. The association of quartz and radiolarians in the same layers may reflect transport from the continental slope by turbidity currents. As in the previous unit, pyrite is common $(2-3 \%)$.

Degree of carbonate dissolution: The preservation of planktonic foraminifers in unit III is very similar to that in unit I. Their fragmentation varies from 5 to 25 per cent and is highest in the darker marly layers rich in pyrite. Post-depositional dissolution might have occured. 


\section{Unit IV}

Mudstone; probably with intercalated sandstone and conglomerate, and perhaps chert. (Cores 415A-1 through 415A-6, Section $1,1-7 \mathrm{~cm}$; 313 to 476.5 meters; lower Eocene to upper Paleocene).

$\mathrm{CaCO}_{3}$ content: About 2.5 per cent.

Sand content: About 1.2 per cent. The sand content is very low, compared to higher units. On the other hand, there are intercalations of pebbles (415A-1).

Sand composition: The sand fraction along Core $415 \mathrm{~A}-1$ is very different from that in other cores. It is essentially a foraminifer (25\% benthic, $20 \%$ planktonic) sand, rich in quartz (10-25\%) and bearing fish debris $(3-7 \%)$ and chert fragments $(10-15 \%)$. No siliceous microfossils are present. The fragmentation of the planktonic foraminifers is close to 30 per cent. The sand fraction of core $415 \mathrm{~A}-5$ is rich in siliceous debris $(<25 \%)$, planktonic foraminifers $(<50 \%)$, and pyrite $(2-30 \%)$.

Degree of carbonate dissolution: Planktonic foraminifers are much better preserved (fragmentation $<5 \%$ ) than in the previous units.

\section{Unit V}

Marlstone with intercalation of nannofossil chalk and limestone (core 415A-6, except for 0 to $7 \mathrm{~cm}$ of Section 1; 476.5 to 491 meters; upper and lower Paleocene).

Carbonate content: 44 to 84 per cent.

Sand content: 0.7 to 2.2 per cent. As for unit IV, the sand content is very low.

Sand composition: Aggregates are abundant (5$9 \%$ ), but they result from induration of the sediment. If we except these aggregates, which in reality belong to the clay fraction, the sand fraction is composed of planktonic foraminifers $(\leq 50 \%)$ associated with benthic foraminifers $(\leq 20 \%)$, radiolarians $(\leq 10 \%)$, quartz $(\leq 10 \%)$, and minor amounts of diatoms $(\leq 1 \%)$, fish debris $(\leq 3 \%)$, glauconite $(\leq 3 \%)$, and echinoids $(\leq 2 \%)$. Pyrite is abundant $(-30 \%)$ in a few levels (e.g., 415A-6-2, 37-39 cm).

The presence of glauconite associated with benthic foraminifers, echinoids, and fish debris suggests that at least part of the sediment was transported by turbidity currents or slumping from the continental slope.

Degree of carbonate dissolution: The degree of fragmentation is very low $(<5 \%)$. Planktonic foraminifers are well preserved. Their apparent abundance decrease is due to dilution and not to dissolution.

\section{Unit VI}

Claystone and dolomitic shale with interbeds of marlstone, dolomite, and limestone (Cores 415A-7 through $415 \mathrm{~A}-15$; 491 to 1041.5 meters; Cenomanian to upper Albian).

\section{Sub-Unit VIa (Core 415A-7; 491-551 m)}

This core can be separated from the rest with respect to its carbonate content, sand-fraction composition, and preservation.

Carbonate content: 3 per cent.

Sand content: 0.1 per cent.
Sand composition: This sand fraction is a typical dissolution residue, with only trace of planktonic foraminifers $(100 \%$ fragmented), but abundant radiolarians $(80 \%)$ and fish debris $(10 \%)$. The terrigenous content is low: 3 per cent quartz and 1 per cent micas.

Degree of carbonate dissolution: The planktonic foraminifers are almost nonexistent and completely fragmented; consequently the sediment of Core 415A-7 might have been deposited at or very close to the CCD. Nevertheless, the abundance of biogenic siliceous particles and of fish debris might reflect relatively high oceanic fertility, which may be partly linked to post-depositional dissolution.

\section{Sub-Unit VIb (Cores 415-8 through 415A-14; 551-1041.5 m)}

Carbonate content: 25 to 56.8 per cent.

Sand content: 0.2 to 9.1 per cent. The fluctuations are still partly related to the presence of abundant (20$60 \%$ ) clay aggregates.

Sand composition: Planktonic foraminifers and radiolarians are alternately dominant in the sand fraction. Planktonic foraminifers vary from 2 to 50 per cent, and radiolarians from 2 to 45 per cent. They are associated with fish debris $(3-20 \%)$, benthic foraminifers $(0.5-5 \%)$ and pyrite (1-3\%). Furthermore, bivalves are present in Cores 12 and 14, where they reach respectively 70 and 10 per cent. They may have been transported from the continental slope and reflect the allochthonous nature of part of the sediment. The sand fraction is devoid of terrigenous particles, except in Core 14, which contains 2 per cent quartz and 1 per cent micas.

Degree of carbonate dissolution: Planktonic foraminifers are well preserved (fragmentation $\leq 2 \%$ ).

\section{Sub-Unit VIc (Cores 415A-15; 1041.5-1079.5 m)}

The only sample taken in this sub-unit contains 18.4 per cent carbonate. It was almost impossible to sieve the sand fraction.

\section{Site 416}

All data are presented on Figure 2 and in Tables 2, 3 and 4.

\section{Unit I}

Nannofossil marl and nannofossil ooze (Cores 416-1, 416A-1-1, 370-1; 0 to about 100 meters; Quaternary to middle Miocene).

$\mathrm{CaCO}_{3}$ content: 30.5 to 73 per cent. The first value characterized the marly, light-olive-gray nannofossil marl, the second one the light-gray nannofossil ooze.

Sand content: 0.1 to 15.6 per cent.

Composition: The sand fraction is essentially constituted of planktonic foraminifers (50-98\%), associated with quartz $(<30 \%)$ and with small amounts of micas $(\leq 2 \%)$, benthic foraminifers $(1-3 \%)$, fish debris $(0.5-$ $1 \%)$, echinoids $(0.5-2 \%)$, pyrite $(<3 \%)$, and glauconite $(\leq 10 \%)$. Aggregates are present in most of the samples and vary from 2 to 30 per cent. 
Degree of carbonate dissolution: The fragmentation of the planktonic foraminifers varies from 5 to 40 per cent, but is close to 25 per cent in most of the samples. That means that these sediments were deposited well above the lysocline.

\section{Unit II}

Graded sequences of sandstone, diatom-rich nannofossil marls, nannofossil marl and chalk (Cores 416A-1$1,90 \mathrm{~cm}$ through $416 \mathrm{~A}-3,370-2$ through $370-7 ; 100$ to 457 meters; early Miocene to late middle Eocene).

$\mathrm{CaCO}_{3}$ content: 2.4 to 79 per cent. The lowest value characterizes the very dark-gray layers, the highest one corresponding to the olive-gray to white nannofossil chalks.

Sand content: 0.1 to 62.2 per cent. The highest values correspond to samples with large amounts (30-70\%) of clayey-quartzose aggregates, where quartz is of silt size and consequently not relevant to this fraction.

Composition: The presence of turbidites throughout this unit explains the large fluctuations of the sand-fraction composition. Dominant components are planktonic foraminifers $(3-90 \%)$ and radiolarians $(1-80 \%)$. They are associated with diatoms $(1-70 \%)$, benthic foraminifers $(1-30 \%)$, echinoids $(1-5 \%)$, fish debris (1$50 \%)$, quartz $(1-20 \%)$, pyrite $(1-75 \%)$, and glauconite (1-10\%).

One of the major points characterizing unit II is the presence and relative abundance of radiolarians and diatoms, which were absent in unit I. They seem to be at least partly transported from the continental slope by turbiditic currents, together with fine quartz, glauconite, echinoids, traces of bivalves, and even with benthic foraminifers. Within the turbiditic sequences, the coarse fraction is mainly hemipelagic and rich in radiolarians, but most poorer in planktonic foraminifers. The decrease of the planktonic-foraminifer population is thus more related to dilution by turbiditic material than to carbonate dissolution.

Another point is the local enrichment of the coarse fraction in pyrite (up to $75 \%$ ), indicating the degree of reduction. Degree of reduction probably is related to the abundance of organic matter in an area of high oceanic productivity, as reflected by the relative abundance of biogenic siliceous particles.

Degree of carbonate dissolution: The degree of fragmentation of the planktonic foraminifers is highly variable $(2-50 \%)$. Fluctuations are probably related partly to the cyclicity of the turbidites and partly to the oceanic fertility. Turbiditic currents transported to the deep basin well preserved planktonic foraminifers. They also rapidly embedded the foraminifers already deposited in the basin, thus protecting them from intensive dissolution. On the other hand, they could also bring from the continental slope organic-matter-rich sediment which might contribute to post-depositional carbonate dissolution.

\section{Unit III}

Turbidities: mudstone, siltstone, sandstone, and gravel, with some porcellanite and dolomitic marlstone
(Cores 416A-4, 416A-5-1, and 3709-8 to the upper part of $370-18 ; 457$ to 642 meters; middle to early Eocene).

As unit III was barely sampled at Site $416 \mathrm{~A}(50 \mathrm{~cm}$ in $416 \mathrm{~A}-4$ and $60 \mathrm{~cm}$ in $416 \mathrm{~A}-5-1$ ), we had to consider the corresponding section at Site 370 .

$\mathrm{CaCO}_{3}$ content: 4.9 to 51.2 per cent. Most of the samples could be classified either as calcareous mud $\left(10 \% \leq \mathrm{CaCO}_{3} \leq 60 \%\right)$. Both types alternate within the turbiditic sequences, but marls are more frequent at the base of the unit (590-642 m).

Sand content: 0.3 to 36.6 per cent. As well as the carbonate content, the coarse fraction content fluctuates highly inside the turbiditic sequences. Furthermore, the presence of abundant clayey-quartzose aggregates (too hard to be sieved) is partly responsible for the apparent fluctuations. This is particularly obvious at Site 370 in Core 17 (Section 2, 137-140 cm), where the sand fraction reaches 51.5 per cent but contains 85 per cent clay aggregates.

Composition: On the basis of the sand fraction composition, unit III is extremely different from unit II. In unit III the sand fraction is essentially of terrigenous origin. If we exclude the aggregates, the quartz content is on the average close to 60 per cent and even reches 90 per cent. It is associated with planktonic foraminifers $(5-60 \%, 25 \%$ average) benthic foraminifers $(2-30 \%)$, radiolarians $(2-10 \%)$, fish debris $(1-6 \%)$, pyrite $(2-25 \%)$, glauconite $(1-5 \%)$, and micas $(\leq 2 \%)$. There are not diatoms in this unit.

Degree of carbonate dissolution: Compared to the planktonic foraminifers of unit II the foraminifers of unit III are better preserved (fragmentation $\leq 20 \%$ on the average). One exception however is Core 16, Section 2,57 to $60 \mathrm{~cm}$, where fragmentation reaches 75 per cent. No obvious reason for this exception appears.

\section{Unit IV}

Nannofossil claystone and marlstone (lower part of Core $370-18$, CC through Core 370-19; 642 to 661 meters; Paleocene).

Unit IV was not receovered at Site 416 and for this reason was defined at Site 370 . The present study of unit IV is based on only one sample $(19-1,116-118 \mathrm{~cm})$.

$\mathrm{CaCO}_{3}$ content: 30.9 per cent.

Sand content: 1 per cent.

Composition: The major difference from the previous unit is the drastic increase in radiolarians $(40 \%)$. They are associated with benthic foraminifers $(15 \%)$, planktonic foraminifers $(10 \%)$, quartz $(10 \%)$, glauconite $(2 \%)$, and fish debris.

\section{Unit V}

Claystone, nannofossil claystone, and silty claystone, with minor siltstone, mudstone, and pebbly mudstone (Cores 416A-5, CC and 416A-6, 370-20 to 370-35; 661 to about 880 meters; Albian to Barremian).

Because of the increase in compaction and induration of the sediment with depth, it was more and more difficult to sieve the sand fraction. From unit V through unit VI it was impossible to continuously analyze the sand fraction. In fact, most of the residue of the sieve consist- 
ed of indurated clayey aggregates embedding quartzose or biogenic particles. No good estimation of the frequency of the sand size particles was possible. Consequently, the values of the sand content given in Table 4 have to be considered with caution.

Unit V was sparsely cored at Site 416. Consequently, it has to be again defined at Site 370 .

Carbonate content: 2.4 per cent.

Sand content: 0.5 to 1.4 per cent.

Composition: The sand fraction is characterized by the absence of biogenic particles, except fish debris $(<7 \%)$, and by the high abundance of pyrite $(0.5-98 \%)$. Quartz is also locally abundant $(2-90 \%)$.

Degree of carbonate dissolution: No foraminifers are present either in the sand fraction or in the finer fraction of the sediment. The absence of calcareous particles in the sediment, excepting a few dolomite rhombs, is probably due to dilution of noncalcareous terrigenous material, rather than to dissolution.

Nannofossils were recovered in claystone in this lithologic unit at Site 370 .

\section{Unit VI}

Distal turbidites: quartzone sandstone, mudstone, claystone, marlstone (Cores 416A-7 to 416A-36-2, 37035 to $370-51 ; 880$ to 1428 meters; Hauterivian to late Valanginian).

$\mathrm{CaCO}_{3}$ content: 2.4 to 65.3 per cent.

Sand content: 0.01 to 50.6 per cent.

Composition: If we except the presence of artificial clayey-quartzose aggregates, the sand fraction throughout this unit is, as in the previous unit, characterized by relatively abundant pyrite $(0.5-100 \%)$ and plant debris $(\leq 60 \%)$. The pyrite constitutes 100 per cent of the sand fraction in Cores 25 (Section 1, 101-102 cm), 29 (Section 5, 12-14 cm), and 30 (Section 3, 57-60 cm). The maxima of plant debris are encountered in Cores 14 (30\% in Section 1, 60\% in core catcher) and $16(40 \%$ in Section 2). Fish debris is rare, except in Core 9 (Section $1,102-104 \mathrm{~cm}$ ), where it reaches 20 per cent.

Degree of carbonate dissolution: Only traces of planktonic foraminifers are present, except in Core 15 where, in Section $1(18-20 \mathrm{~cm})$ foraminifers constitute
10 per cent of the sand fraction. As in the previous unit, the scarcity of planktonic foraminifers is probably due to dilution, rather than to dissolution.

\section{Unit VII}

Turbidites: alternating quartzose siltstone and mudstone cycles with hard micrite and calcarenite (Cores 416A-36-3 through 416A-57; 1428 to 1624 meters; early Valanginian to Tithonian).

This unit was not cored at Site 370 .

$\mathrm{CaCO}_{3}$ content: 3.3 to 87 per cent.

Sand content: 0.02 to 6.7 per cent.

Composition: Throughout this unit, it was almost impossible to sieve the sand fraction from the sediment. All the residues obtained on the $63-\mu \mathrm{m}$ sieve consist of artificial clayey aggregates, locally containing quartz, micas, and pyrite. No biogenic particles are visible.

Degree of carbonate dissolution: The selected samples yield no information about carbonate dissolution. The presence of carbonate cycles with micritic limestone throughout this unit seems however to reflect a low degree of dissolution, even if the carbonate-rich sediment could have been rapidly buried (and thus protected from eventual dissolution) under terrigenous turbiditic sequences.

\section{REFERENCES}

Adelseck, C. G., Jr., 1977. Recent and late Pleistocene sediments from the eastern equatorial Pacific Ocean: sedimentation and dissolution, Ph.D. Thesis, University of California, San Diego.

Berger, W. H., 1970a. Planktonic foraminifers-selective solution with the lysocline. Mar. Geol., v. 8, p. 111-138. 1970b. Biogenous deep-sea sediments: fractionation by deep-sea circulation. Geol. Soc. Am. Bull., v. 81, p. 1385-1402.

Melguen, M., and Thiede, J., 1974. Facies distribution and dissolution depths of surface sediment components from the Vema channel and the Rio Grande Rise (southwest Atlantic ocean). Mar. Geol., v. 17, p. 341-353.

Terry, R. D., and Chillingar, G. V., 1955. Charts for estimating percentage composition of rocks and sediments. J. Sediment. Petrol., v. 25, p. 229-234. 